\title{
ManUel JoÃo Ramos - Of Hairy KinGs AND SAINTLY SLAVES. AN ETHIOPIAN TRAVELOGUE [RECENSION]
}

Anaïs Wion ${ }^{1}$

CNRS, Ivry-sur-Seine, France

Cet ouvrage est une version modifiée de Historias Etiopies. Diario de viagem du même auteur publié en 2000 chez Assirio \& Alvim à Lisbonne. On y retrouve les trois fils conducteurs qui rendent compte du premier voyage de l'anthropologue portugais Manuel Joao Ramos sur les hauts plateaux éthiopiens, en 1999, il y a vingt années de cela : le dessin ; le récit de voyage ; la collecte de traditions orales. Le choix de l'Éthiopie comme destination en 1999 n'était pas le fruit du hasard, puisque Manuel João Ramos avait alors déjà travaillé sur le mythe chrétien du Prêtre Jean, ce roi-prêtre oriental qui dès la fin du Moyen-Âge devait permettre au monde chrétien de réaliser son unité au détriment des puissances musulmanes. Poursuivant ces recherches sur les terres éthiopiennes, ses enquêtes interrogent en premier lieu la mémoire de la présence portugaise dans les régions où les armées portugaises puis les jésuites furent présents au XVI et au début du XVII siècle.

Découvrant l'Éthiopie, il dessine, il note, il enregistre l'histoire orale. Ce sont ces trois pratiques de son métier d'anthropologue qu'il juxtapose ici, sans qu'aucun de ces trois indices de son passage par les hautes terres éthiopiennes ne se fasse totalement l'illustration des autres mais au contraire, chacun existant pleinement pour lui-même.

Examinons d'abord les dessins, de vivants croquis au feutre noir parfois rehaussés d'aquarelle. Manuel Ramos n'est pas un dessinateur mais plutôt quelqu'un qui « n'a jamais cessé de dessiner », ou même plus, qui " n'a pas abandonné le dessin pour l'écriture » comme il le dit luimême. Il dessine pour voir autrement, pour être présent dans l'instant, pour ralentir le temps du voyage, pour ne pas prendre des images mais pour les faire. Ce geste graphique n'a d'autre fonction que de permettre d'exister. Ses carnets ne sont donc pas destinés à servir de mémoire ou de ressources documentaires mais ils accomplissent leur rôle au moment et uniquement au moment où ils sont réalisés. Cette première publication en 2000, si elle avait changé le statut de ses dessins et ouvert la voie à d'autres publications scientifiques les intégrant pleinement, n'a en

1 Institut des Mondes Africains. Contact de l'auteur : anais.wion@univ-paris1.fr. Le livre de Manuel João Ramos a été publié pour Sean Kingston Publishing en 2018 (ISBN 978-1-907774-19-5, traduction par Christopher Tribe). 
rien modifié ses pratiques. ${ }^{2}$ Faisant confiance à son cerveau autant qu'à ses mains pour choisir ce qui est essentiel, il croque tout autant l'exotique (tatouages des femmes amhara, chars blindés abandonnés en pleine ville) que le banal (scènes de rue ou de bord de route), les paysages grandioses tout autant que les bâtiments modernes ou historiques, les rencontres diverses. Se dessinant parfois lui-même en train de dessiner, l'anthropologue encore ignorant du pays où il s'aventure s'approche au plus près du non verbal.

S'imprégnant de ce qui l'entoure, il recopie les enseignes rédigées avec les signes de l'alpha-syllabaire éthiopien, tire le portrait des informateurs en précisant leurs noms, la date, les lieux des rencontres, note les mots essentiels aux échanges quotidiens en amharique. Des références bibliographiques, très datées aujourd'hui ( $\mathrm{S}$. Kaplan était encore une référence majeure pour comprendre le monachisme éthiopien), nous font autant voyager dans le temps que la vue de la place principale de Gondar ornée d'un monument communiste datant du Derg là où aujourd'hui trône une statue du roi Tewodros. Ce premier carnet de voyage éthiopien nous donne véritablement à voir l'anthropologue découvrant un terrain d'étude. D'autres dessins publiés ultérieurement montrent le pas franchi depuis ce premier terrain (notamment pour ce qui est de Gondar, on peut comparer avec le City Guide of Gondar, publié en 2014, dans lequel le regard semble plus apaisé, moins stupéfait par l'abondance de détails visuels nouveaux). ${ }^{3}$

Intercalé avec des pages entières du carnet de dessin, vient le texte d'un «Ethiopian travelogue » daté du jour du départ, le 3 mars, jusqu'au retour 3 mois plus tard à Lisbonne, après avoir réalisé plusieurs périples à Harrar, Gondar, Lalibela, au lac Tana, dans le Godjam. Rédigées sur un ton incisif et souvent ironique, ces pages furent pour certaines immédiatement envoyées pour publication à un journal portugais. Elles narrent la violence du conflit alors en cours avec l'Érythrée, la disparition des juifs éthiopien « rapatriés » en Israël depuis plus de 30 ans mais dont certains sont toujours parqués dans un camp en plein cœur d'Addis Abeba (p. 43), l'intense spiritualité de la semaine de la Passion du Christ suivie de banquets dantesques des fêtes de Pâques, une conversation sur les enjeux de la conservation du patrimoine avec le directeur de l'autorité ad hoc au ministère de la Culture, ou encore le traumatisme de la Terreur Rouge à Gondar (1977-78) et sa probable influence sur la façon dont l'histoire orale est dite aujourd'hui.

Cela m’amène à la troisième partie de l'ouvrage, intitulé « Ethiopian Stories », profondément remaniée par rapport à l'édition originale : le choix des textes oraux a été renouvelé et chaque entretien est accompagné d'une introduction présentant l'informateur, les conditions dans lesquelles ont été faites l'entretien ainsi qu'une analyse rapide du contenu de l'entretien. Les questions posées par l'anthropologue n'apparaissent pas ce qui contraste avec les autres parties de l'ouvrage dans lesquelles au contraire la réflexivité fait la part belle à la présence du chercheur et aux possibles filtres et diffractions que sa présence génère. Si un montage vidéo de 16 minutes de certains entretiens est disponible sur youtube et sur le dépôt d'auto-archivage de l'ISCTE, celui-ci fait peu apparaître le rôle de l'interviewer et celui de ses traducteurs, décontextualisant la parole des historiens éthiopiens. ${ }^{4}$ On voudrait pouvoir accéder à la totalité

2 Ramos, Manuel João. 2015. «Stop the Academic World, I Wanna Get Off in the Quai de Branly. Of sketchbooks, museums and anthropology ", Cadernos de Arte e Antropologia, 4(2) : 53-90.

3 Wion, Anaïs, Sisay Sahile, Ramos, Manuel João. 2014. Etbiopian City Guides: Gondar. Addis Ababa, French Center for Ethiopian Studies ed (https://www.academia.edu/15685307/Gondar_City_Guide, accédé le 26/02/19).

4 Ces vidéos sont acessibles ici : https://www.youtube.com/watch?v=OFxegBMXa1Q\&feature=youtu.be et là : https://repositorio.iscte-iul.pt/handle/10071/16501 (accédés le 26/02/19). 
des entretiens car ce savoir est fragile et les collectes opérées en 1999 par Manuel João Ramos précieuses. On peut dans cet ordre d'idées regretter aussi le manque d'apparat critique puisque le sommaire ne permet pas de naviguer dans l'ouvrage et qu'il n'y a pas d'index. Certes, la publication de ces textes d'histoire orale n'a pas pour objectif de fournir un corpus constitué. Le découpage d'un même entretien pour structurer la narration de façon thématique est un choix antithétique d'une édition de corpus mais sert par contre l'analyse symbolique en permettant des rapprochements de motifs narratifs.

Manuel João Ramos a ainsi collecté la tradition orale auprès d'intellectuels religieux et laïcs de la région de Gondar (quatre intellectuels chrétiens, un musulman et un qemant dans la ville de Gondar ; deux prêtres à Delgi et Danqaz, enfin des paysans et des laïcs à Danqaz, Gorgora, Enfraz et Grañ Bar). Il interroge ses informateurs à propos de la présence portugaise, que ce soit l'envoi de soldats portugais sous la conduite de Christophe da Gama au début du $\mathrm{XVI}^{\mathrm{e}}$ siècle pour aider la couronne éthiopienne à faire face à une guerre de jihad menée par l'imam Ahmed, ou, plus tard, la présence de jésuites portugais qui obtiennent la conversion du roi puis de l'État éthiopien au catholicisme (autour de 1620), jusqu'à l'abdication du roi catholique en 1632 et finalement, l'expulsion du dernier père portugais dans la seconde moitié du $\mathrm{XVII}^{\mathrm{e}}$ siècle. Si on devine que les questions font démarrer les entretiens sur ce thème chaque entretien se déroule ensuite au gré des connaissances de l'interviewé et de nombreux points sont abordés : la construction des châteaux, des églises et des ponts dans la région du Dambya, la démesure des souverains tuant une femme chaque nuit pour cacher leur animalité, les meurtres de moines perpétrés par Fasiladas (1632-67) et les rivières de sang baignant la fondation de Gondar, les prophéties entourant l'accès au trône de Susenyos (1607-32), etc.

Découvrant au fur et à mesure de ses enquêtes les aspects labiles tout autant que les grands thèmes structurants d'une histoire orale fortement constituée, l'anthropologue veut rendre intelligibles les systèmes symboliques à l'œuvre au travers de ces récits. Il identifie ainsi un premier motif, celui de l'hyper-exogamie, opérant dans plusieurs récits, qui légitime des changements drastiques de dynastie, de religion ou de lieu de pouvoir. Cette grille d'analyse permet de comprendre, par exemple, le motif du mariage, planifié mais non réalisé, du roi Susenyos avec la princesse romaine Zeliha, qui se reporte alors sur son fils, assurant ainsi la continuité successorale et validant, a posteriori, la légitimité sur le trône du roi catholique autant qu'elle entache le règne violent bien qu'orthodoxe de son fils, Fasiladas. Le modèle premier de ce motif de l'hyper-exogamie serait celui de la reine de Saba et du roi Salomon, mythe politique primordial de la royauté éthiopienne chrétienne à travers le texte du Kebra Nagast (Gloire des Rois). Si cette filiation entre le Kebra Nagast et ces thèmes de la tradition orale est possible mais demanderait à être travaillée, Manuel João Ramos frôle peut-être la surinterprétation lorsqu'il propose une analogie entre l'icône royale du Kwerata Re'esu -Ecce Homo servant d'emblème de pouvoir-, et l'Arche d'Alliance (p. 97) ou encore qu'à plusieurs reprises il qualifie Gondar de Nouvelle Jérusalem (p. 143 par ex.). D'autres motifs d'hyper-exogamie traversent les traditions orales éthiopiennes et demanderaient, c'est certain, à être comparés.

Un autre motif récurrent identifié par Manuel João Ramos est celui des figures marginales, telles les esclaves pieuses, les magiciens et les ermites, ou encore les musulmans ou les portugais, figures ambiguës à la fois craintes et méprisées, qui entrent en interaction avec le souverain à des moments critiques et permettent de refonder un cycle religieux ou politique. Ces figures permettent de restaurer l'ordre, d'accompagner vers la résolution d'un déséquilibre le pouvoir politique en calmant son hubris et en le légitimant (voir le résumé p. 180). Ces catégories 
fluides et ambiguës (p. 79) entre le bien et le mal, le haut et le bas de l'échelle sociale et spirituelle donnent son titre à l'ouvrage.

Une autre avancée de cette analyse est de montrer que les prophéties entourant la naissance de Susenyos et le meurtre évité du nourrisson peuvent se comprendre dans un contexte narratif très large, incluant le désordre introduit par la religion catholique. ${ }^{5}$ Ainsi si Susenyos est bien un enfant bâtard promis à régner et qu'il accomplit cette prophétie, sa conversion à la religion catholique agit comme un mauvais sort et le prive du langage en déformant sa langue, une maladie que seule son abdication permet de résoudre. Le pouvoir passe aux mains de son fils, Fasiladas, qui hériterait de son père le mariage avec une princesse romaine mentionné ci-dessus, ainsi qu'une tare physique et mentale, la honte d'une extrême pilosité qui l'amène à perpétrer chaque nuit le meurtre de la femme avec qui il a eu des rapports sexuels.

Pour aussi convaincants que soient certains aspects de cette remise en ordre de la narration, il faut néanmoins prendre garde à l'effet téléologique que peut produire la réorganisation des textes oraux par thèmes et par ordre chronologique. En effet, si les histoires liées au roi Fasiladas et à celle de son père Susenyos partagent des motifs narratifs communs, rien ne permet de dire que ceux-ci ne furent pas initialement dévolus à Fasiladas, puis associés à son père Susenyos. Or Manuel João Ramos postule systématiquement que ceux-ci ont migré de l'histoire du père vers celle du fils, ce qui ne va pas de soi.

Ces quelques mises en garde viennent bien évidemment de mon regard d'historienne sur un matériau que j’ai moi-même considéré de façon très différente, mais je salue avant tout la finesse et l'inventivité des analyses novatrices proposées par Manuel João Ramos.

Pour synthétiser, cet ouvrage reflète plusieurs histoires, celle d'un anthropologue dessinateur immergé pour la première fois dans la réalité éthiopienne et cherchant à remonter le temps et à sonder la mémoire collective ; celle d'un moment particulier pendant lequel l'Éthiopie d'après le Derg et de Meles Zenawi n'a pas encore complètement basculé dans l'ultra libéralisme et le fédéralisme ethnique ; enfin celle liée à la fondation d'une dynastie et d'une capitale au nord du lac Tana, par des souverains puissants et controversés à l'époque moderne.

5 Ce que je n'avais pas fait dans mon analyse d'un corpus semblable, où je m'étais par contre plus attachée à comprendre les mécanismes de construction de ces récits prophétiques et d'en analyser les versions, parfois très différentes, voir Wion, Anaïs. Paradis pour une reine, Paris, Publications de la Sorbonne, 2012. 